\title{
Common fixed points for pointwise Lipschitzian semigroups in modular function spaces
}

\author{
Buthinah A Bin Dehaish ${ }^{1 *}$, Mohamed A Khamsi ${ }^{2,3}$ and Wojciech M Kozlowski ${ }^{4}$
}

\section{"Correspondence:}

bbindehaish@yahoo.com

'Department of Mathematics, King

Abdulaziz University, P.O. Box 53909,

Jeddah, 21593, Saudi Arabia

Full list of author information is

available at the end of the article

\section{第 Springer}

\begin{abstract}
Let $C$ be a $\rho$-bounded, $\rho$-closed, convex subset of a modular function space $L_{\rho}$. We investigate the existence of common fixed points for asymptotic pointwise nonexpansive semigroups of nonlinear mappings $T_{t}: C \rightarrow C$, i.e. a family such that $T_{0}(f)=f, T_{s+t}(f)=T_{s} \circ T_{t}(f)$ and$$
\rho(T(f)-T(g)) \leq \alpha_{t}(f) \rho(f-g),
$$

where $\lim \sup _{t \rightarrow \infty} \alpha_{t}(f) \leq 1$ for every $f \in C$. In particular, we prove that if $L_{\rho}$ is uniformly convex, then the common fixed point is nonempty $\rho$-closed and convex. MSC: Primary 47H09; secondary 46B20; 47H10; 47E10

Keywords: fixed point; modular function space; nonexpansive mapping; Orlicz space; pointwise Lipschitzian mapping; pointwise nonexpansive mapping; semigroup; uniform convexity
\end{abstract}

\section{Introduction}

The purpose of this paper is to prove the existence of common fixed points for semigroups of nonlinear mappings acting in modular function spaces which are natural generalizations of both function and sequence variants of many important, from applications perspective, spaces like Lebesgue, Orlicz, Musielak-Orlicz, Lorentz, Orlicz-Lorentz, Calderon-Lozanovskii spaces and many others, see the book by Kozlowski [1] for an extensive list of examples and special cases. Earlier studies of fixed point theory in modular function spaces can be found in [2-4], see also [5]. Recently, Khamsi and Kozlowski presented a series of fixed point results for pointwise contractions, asymptotic pointwise contractions, pointwise nonexpansive and asymptotic pointwise nonexpansive mappings acting in modular functions spaces [6,7] (all these should be considered in the modular sense, not in the sense of the corresponding norms). These results are also new and of a big interest, even in a much simpler context of 'plain' modular contractions and nonexpansive mappings, i.e., without any pointwise and asymptotic complications.

In many cases, modular type conditions are much more natural as modular type assumptions can be more easily verified than their metric or norm counterparts. Furthermore, there are also important results that can be proved only using the apparatus of modular function spaces. Khamsi et al. demonstrated in [2] that a mapping $T$ is norm-

(c) 2013 Bin Dehaish et al.: licensee Springer. This is an Open Access article distributed under the terms of the Creative Commons Attribution License (http://creativecommons.org/licenses/by/2.0), which permits unrestricted use, distribution, and reproduction in any medium, provided the original work is properly cited. 
nonexpansive in a modular function space $L_{\rho}$ if and only if

$$
\rho(\lambda(T(f)-T(g))) \leq \rho(\lambda(f-g)) \text { for any } \lambda \geq 0 .
$$

They also gave an example of a mapping which is $\rho$-nonexpansive, but it is not normnonexpansive. From this perspective, the fixed point theory in modular function spaces should be considered as complementary to the fixed point theory in normed spaces and in metric spaces.

Let us recall that a family $\left\{T_{t}\right\}_{t \geq 0}$ of mappings forms a semigroup if $T_{0}(x)=x$ and $T_{s+t}=T_{s} \circ T_{t}$, see Definition 2.6 below for details. Such a situation is quite typical in mathematics and applications. For instance, in the theory of dynamical systems, the modular function space $L_{\rho}$ would define the state space and the mapping $(t, f) \rightarrow T_{t}(f)$ would represent the evolution function of a dynamical system. The question about the existence of common fixed points and about the structure of the set of common fixed points, can be interpreted as a question whether there exist points that are fixed during the state space transformation $T_{t}$ at any given point of time $t$, and if yes, what the structure of a set of such points may look like. In the setting of this paper, the state space may be an infinite dimensional vector space. Therefore, it is natural to apply these results not only to deterministic dynamical systems but also to stochastic dynamical systems.

The existence of common fixed points for families of contractions and nonexpansive mappings in the Banach spaces have been investigated since the early 1960s, see, e.g., Belluce and Kirk [8, 9], Browder [10], Bruck [11], DeMarr [12], Lim [13]. The asymptotic approach for finding common fixed points of semigroups of Lipschitzian (but not pointwise Lipschitzian) mappings has also been investigated for some time, see, e.g., Tan and $\mathrm{Xu}$ [14]. It is worthwhile mentioning the recent studies on the special case, when the parameter set for the semigroup is equal to $\{0,1,2,3, \ldots\}$ and $T_{n}=T^{n}$, the $n$th iterate of an asymptotic pointwise nonexpansive mapping, i.e., $T: C \rightarrow C$ such that there exists a sequence of functions $\alpha_{n}: C \rightarrow[0, \infty)$ with

$$
\left\|T^{n}(f)-T^{n}(g)\right\| \leq \alpha_{n}(f)\|f-g\| \quad \text { for any } f, g \in C
$$

and $\lim \sup _{n \rightarrow \infty} \alpha_{n}(f)=1$ for any $f \in C$. Kirk and Xu [15] proved the existence of fixed points for asymptotic pointwise contractions and asymptotic pointwise nonexpansive mappings in the Banach spaces, while Hussain and Khamsi extended this result to metric spaces [16] and Khamsi and Kozlowski to modular function spaces [6, 7]. Kozlowski in [17] and [18] proved convergence to fixed points of some iterative algorithms, applied to asymptotic pointwise nonexpansive mappings in the Banach spaces, and the existence of common fixed points of semigroups of asymptotic pointwise nonexpansive semigroups in the Banach spaces [19]. Convergence of generalized Mann and Ishikawa algorithms to common points of such semigroups in Banach spaces was established in [20] and [21]. In the context of modular function spaces, convergence to fixed points of some iterative algorithms, applied to asymptotic pointwise nonexpansive mappings, was proven by Bin Dehaish and Kozlowski in [22].

In this paper, we extend the definition of asymptotic pointwise nonexpansive mappings to semigroups of mappings and prove some common fixed point results in the context of modular function spaces. Therefore, our results generalize the results of Kozlowski [23], who proved the existence of common fixed points for semigroups of nonexpansive 
mappings in modular functions spaces, to the pointwise asymptotic semigroups. However, methods used in the current paper are substantially different due to the asymptotic behavior of semigroups in question. It is worth noting that existence of semigroups of nonexpansive mappings in modular function spaces was discussed by Khamsi [24] in the context of Musielak-Orlicz spaces and discussed applications to differential equations.

\section{Preliminaries}

Let us introduce basic notions related to modular function spaces and related notation, which will be used in this paper. For further details, we refer the reader to preliminary sections of the recent articles $[6,7,22]$ or to the survey article [5], see also $[1,25,26]$ for the standard framework of modular function spaces.

Let $\Omega$ be a nonempty set, and let $\Sigma$ be a nontrivial $\sigma$-algebra of subsets of $\Omega$. Let $\mathcal{P}$ be a $\delta$-ring of subsets of $\Omega$ such that $E \cap A \in \mathcal{P}$ for any $E \in \mathcal{P}$ and $A \in \Sigma$. Let us assume that there exists an increasing sequence of sets $K_{n} \in \mathcal{P}$ such that $\Omega=\bigcup K_{n}$. By $\mathcal{E}$ we denote the linear space of all simple functions with supports from $\mathcal{P}$. By $\mathcal{M}_{\infty}$ we will denote the space of all extended measurable functions, i.e., all functions $f: \Omega \rightarrow[-\infty, \infty]$ such that there exists a sequence $\left\{g_{n}\right\} \subset \mathcal{E},\left|g_{n}\right| \leq|f|$ and $g_{n}(\omega) \rightarrow f(\omega)$ for all $\omega \in \Omega$. By $1_{A}$ we denote the characteristic function of the set $A$.

Definition 2.1 [1] Let $\rho: \mathcal{M}_{\infty} \rightarrow[0, \infty]$ be a nontrivial, convex and even function. We say that $\rho$ is a regular convex function pseudomodular if:

(i) $\rho(0)=0$;

(ii) $\rho$ is monotone, i.e., $|f(\omega)| \leq|g(\omega)|$ for all $\omega \in \Omega$ implies $\rho(f) \leq \rho(g)$, where $f, g \in \mathcal{M}_{\infty}$;

(iii) $\rho$ is orthogonally subadditive, i.e., $\rho\left(f 1_{A \cup B}\right) \leq \rho\left(f 1_{A}\right)+\rho\left(f 1_{B}\right)$ for any $A, B \in \Sigma$ such that $A \cap B \neq \emptyset, f \in \mathcal{M}$;

(iv) $\rho$ has the Fatou property, i.e., $\left|f_{n}(\omega)\right| \uparrow|f(\omega)|$ for all $\omega \in \Omega$ implies $\rho\left(f_{n}\right) \uparrow \rho(f)$, where $f \in \mathcal{M}_{\infty}$;

(v) $\rho$ is order continuous in $\mathcal{E}$, i.e., $g_{n} \in \mathcal{E}$ and $\left|g_{n}(\omega)\right| \downarrow 0$ implies $\rho\left(g_{n}\right) \downarrow 0$.

Similarly, as in the case of measure spaces, we say that a set $A \in \Sigma$ is $\rho$-null if $\rho\left(g 1_{A}\right)=0$ for every $g \in \mathcal{E}$. We say that a property holds $\rho$-almost everywhere if the exceptional set is $\rho$-null. As usual, we identify any pair of measurable sets, whose symmetric difference is $\rho$-null, as well as any pair of measurable functions, differing only on a $\rho$-null set. With this in mind, we define $\mathcal{M}=\left\{f \in \mathcal{M}_{\infty} ;|f(\omega)|<\infty \rho\right.$-a.e. $\}$, where each element is actually an equivalence class of functions equal $\rho$-a.e. rather than an individual function.

Definition 2.2 [1] We say that a regular function pseudomodular $\rho$ is a regular convex function modular if $\rho(f)=0$ implies $f=0 \rho$-a.e. The class of all nonzero regular convex function modulars, defined on $\Omega$ will be denoted by $\Re$.

Definition 2.3 $[1,25,26]$ Let $\rho$ be a convex function modular. A modular function space is the vector space $L_{\rho}=\{f \in \mathcal{M} ; \rho(\lambda f) \rightarrow 0$ as $\lambda \rightarrow 0\}$. In the vector space $L_{\rho}$, the following formula

$$
\|f\|_{\rho}=\inf \left\{\alpha>0 ; \rho\left(\frac{f}{\alpha}\right) \leq 1\right\}
$$

defines a norm, frequently called Luxembourg norm. 
The following notions will be used throughout the paper.

Definition 2.4 [2] Let $\rho \in \Re$.

(a) We say that $\left\{f_{n}\right\}$ is $\rho$-convergent to $f$ and write $f_{n} \rightarrow f(\rho)$ if and only if $\rho\left(f_{n}-f\right) \rightarrow 0$.

(b) A sequence $\left\{f_{n}\right\}$, where $f_{n} \in L_{\rho}$, is called $\rho$-Cauchy if $\rho\left(f_{n}-f_{m}\right) \rightarrow 0$ as $n, m \rightarrow \infty$.

(c) We say that $L_{\rho}$ is $\rho$-complete if and only if any $\rho$-Cauchy sequence in $L_{\rho}$ is $\rho$-convergent.

(d) A set $B \subset L_{\rho}$ is called $\rho$-closed if for any sequence of $f_{n} \in B$, the convergence $f_{n} \rightarrow f(\rho)$ implies that $f$ belongs to $B$.

(e) A set $B \subset L_{\rho}$ is called $\rho$-bounded if $\sup \{\rho(f-g) ; f \in B, g \in B\}<\infty$.

Since $\rho$ fails in general the triangle identity, many of the known properties of limit may not extend to the $\rho$-convergence. For example, the $\rho$-convergence does not necessarily imply the $\rho$-Cauchy condition. However, it is important to remember that the $\rho$-limit is unique when it exists. The following proposition brings together few facts that will be often used in the proofs of our results.

Proposition 2.1 [1] Let $\rho \in \Re$.

(i) $L_{\rho}$ is $\rho$-complete.

(ii) $\rho$-balls $B_{\rho}(f, r)=\left\{g \in L_{\rho} ; \rho(f-g) \leq r\right\}$ are $\rho$-closed.

(iii) If $\rho\left(\alpha f_{n}\right) \rightarrow 0$ for an $\alpha>0$, then there exists a subsequence $\left\{g_{n}\right\}$ of $\left\{f_{n}\right\}$ such that $g_{n} \rightarrow 0 \rho$-a.e.

(iv) $\rho(f) \leq \liminf _{n \rightarrow \infty} \rho\left(f_{n}\right)$, whenever $f_{n} \rightarrow f \rho$-a.e. (Note: this property is equivalent to the Fatou property.)

Let us recall the definition of an asymptotic pointwise nonexpansive mapping acting in a modular function space.

Definition 2.5 [7] Let $\rho \in \Re$, and let $C \subset L_{\rho}$ be nonempty and $\rho$-closed. A mapping $T$ : $C \rightarrow C$ is called

(i) a pointwise Lipschitzian mapping, if there exists $\alpha: C \rightarrow[0, \infty)$ such that

$$
\rho(T(f)-T(g)) \leq \alpha(f) \rho(f-g) \quad \text { for any } f, g \in C ;
$$

(ii) an asymptotic pointwise nonexpansive, if there exists a sequence of mappings $\alpha_{n}: C \rightarrow[0, \infty)$ such that

$$
\rho\left(T^{n}(f)-T^{n}(g)\right) \leq \alpha_{n}(f) \rho(f-g) \quad \text { for any } f, g \in C
$$

and $\lim \sup _{n \rightarrow \infty} \alpha_{n}(f) \leq 1$ for any $f \in L_{\rho}$.

A point $f \in C$ is called a fixed point of $T$, whenever $T(f)=f$. The set of fixed points of $T$ will be denoted by $F(T)$.

This definition is now extended to a one-parameter family of mappings.

Definition 2.6 A one-parameter family $\mathcal{F}=\left\{T_{t}: t \geq 0\right\}$ of mappings from $C$ into itself is said to be a asymptotic pointwise nonexpansive semigroup on $C$ if $\mathcal{F}$ satisfies the following conditions: 
(i) $T_{0}(f)=f$ for $f \in C$;

(ii) $T_{t+s}(f)=T_{t}\left(T_{s}(f)\right)$ for $f \in C$ and $t, s \in[0, \infty)$;

(iii) for each $t \geq 0, T_{t}$ is an asymptotic pointwise nonexpansive mapping, i.e., there exists a function $\alpha_{t}: C \rightarrow[0, \infty)$ such that

$$
\rho\left(T_{t}(f)-T_{t}(g)\right) \leq \alpha_{t}(f) \rho(f-g) \quad \text { for all } f, g \in C
$$

such that $\limsup _{t \rightarrow \infty} \alpha_{t}(f) \leq 1$ for every $f \in C$;

(iv) for each $f \in C$, the mapping $t \rightarrow T_{t}(f)$ is $\rho$-continuous.

For each $t \geq 0$, let $F\left(T_{t}\right)$ denote the set of its fixed points. Define then the set of all common fixed points set for mappings from $\mathcal{F}$ as the following intersection

$$
F(\mathcal{F})=\bigcap_{t \geq 0} F\left(T_{t}\right) .
$$

Note that without loss of generality, we may assume $\alpha_{t}(f) \geq 1$ for any $t \geq 0$ and $f \in C$, and $\lim \sup _{t \rightarrow \infty} \alpha_{t}(f)=\lim _{t \rightarrow \infty} \alpha_{t}(f)=1$.

\section{Existence of common fixed points}

The concept $\rho$-type is a powerful technical tool, which is used in the proofs of many fixed point results. The definition of a $\rho$-type is based on a given sequence. In this work, we generalize this definition to be adapted to one-parameter family of mappings.

Definition 3.1 Let $K \subset L_{\rho}$ be convex and $\rho$-bounded.

(1) A function $\tau: K \rightarrow[0, \infty]$ is called a $\rho$-type (or shortly a type) if there exists a one-parameter family $\left\{h_{t}\right\}_{t \geq 0}$ of elements of $K$ such that for any $f \in K$ there holds

$$
\tau(f)=\inf _{M>0}\left(\sup _{t \geq M} \rho\left(h_{t}-f\right)\right) .
$$

(2) Let $\tau$ be a type. A sequence $\left\{g_{n}\right\}$ is called a minimizing sequence of $\tau$ if

$$
\lim _{n \rightarrow \infty} \tau\left(g_{n}\right)=\inf \{\tau(f): f \in K\} .
$$

Note that $\tau$ is convex, provided $\rho$ is convex.

Let us recall the modular equivalents of uniform convexity introduced in [7].

Definition 3.2 Let $\rho \in \Re$. We define the following uniform convexity (UC) type properties of the function modular $\rho$ :

(i) Let $r>0, \varepsilon>0$. Define

$$
D(r, \varepsilon)=\left\{(f, g): f, g \in L_{\rho}, \rho(f) \leq r, \rho(g) \leq r, \rho(f-g) \geq \varepsilon r\right\} .
$$

Let

$$
\delta(r, \varepsilon)=\inf \left\{1-\frac{1}{r} \rho\left(\frac{f+g}{2}\right):(f, g) \in D(r, \varepsilon)\right\} \quad \text { if } D(r, \varepsilon) \neq \emptyset,
$$

and $\delta(r, \varepsilon)=1$ if $D(r, \varepsilon)=\emptyset$. We say that $\rho$ satisfies (UC) if for every $r>0, \varepsilon>0$, $\delta(r, \varepsilon)>0$. Note that for every $r>0, D(r, \varepsilon) \neq \emptyset$, for $\varepsilon>0$ small enough. 
(ii) We say that $\rho$ satisfies (UUC) if there exists $\eta(s, \varepsilon)>0$, for every $s \geq 0$, and $\varepsilon>0$ such that

$$
\delta(r, \varepsilon)>\eta(s, \varepsilon)>0 \quad \text { for } r>s .
$$

The following technical lemma is very useful throughout this paper (see [7] for its proof).

Lemma 3.1 Let $\rho \in \Re$ be $(U U C)$. Let $R>0$. Assume that $\left\{f_{n}\right\}$ and $\left\{g_{n}\right\}$ are in $L_{\rho}$ such that

$$
\limsup _{n \rightarrow \infty} \rho\left(f_{n}\right) \leq R ; \quad \limsup _{n \rightarrow \infty} \rho\left(g_{n}\right) \leq R \quad \text { and } \quad \lim _{n \rightarrow \infty} \rho\left(\frac{f_{n}+g_{n}}{2}\right)=R .
$$

Then we must have $\lim _{n \rightarrow \infty} \rho\left(f_{n}-g_{n}\right)=0$.

The following property plays in the theory of modular function space a role similar to the reflexivity in the Banach spaces, see, e.g., [3].

Definition 3.3 We say that $L_{\rho}$ has property $(R)$ if and only if every nonincreasing sequence $\left\{C_{n}\right\}$ of nonempty, $\rho$-bounded, $\rho$-closed and convex subsets of $L_{\rho}$ has a nonempty intersection.

Similarly as in the Banach space case, the modular uniform convexity implies the property $(R)$.

Theorem 3.1 [7] Let $\rho \in \Re$ be (UUC), then $L_{\rho}$ has a property $(R)$.

The next lemma is the generalization of the minimizing sequence property for types defined by the sequences in Lemma 4.3 in [6] to the one-parameter semigroup case.

Lemma 3.2 Assume $\rho \in \Re$ is (UUC). Let $C$ be a nonempty, $\rho$-bounded, $\rho$-closed and convex subset of $L_{\rho}$. Let $\tau$ be a type defined by a one-parameter family $\left\{h_{t}\right\}_{t \geq 0}$ in $C$.

(i) If $\tau\left(f_{1}\right)=\tau\left(f_{2}\right)=\inf _{f \in C} \tau(f)$, then $f_{1}=f_{2}$.

(ii) Any minimizing sequence $\left\{f_{n}\right\}$ of $\tau$ is $\rho$-convergent. Moreover, the $\rho$-limit of $\left\{f_{n}\right\}$ is independent of the minimizing sequence.

Proof First, let us prove (i). Let $f_{1}, f_{2} \in C$ such that $\tau\left(f_{1}\right)=\tau\left(f_{2}\right)=\inf _{f \in C} \tau(f)$. Let us consider two cases.

Case 1: $\inf _{f \in C} \tau(f)=0$. Since

$$
\rho\left(\frac{f_{1}-f_{2}}{2}\right)=\rho\left(\frac{f_{1}-h_{t}+h_{t}-f_{2}}{2}\right) \leq \rho\left(f_{1}-h_{t}\right)+\rho\left(h_{t}-f_{2}\right)
$$

for any $t \geq 0$, we get

$$
\rho\left(\frac{f_{1}-f_{2}}{2}\right) \leq \sup _{t \geq M} \rho\left(f_{1}-h_{t}\right)+\sup _{t \geq M} \rho\left(h_{t}-f_{2}\right)
$$


for any $M>0$. Since

$$
\tau(f)=\inf _{M>0}\left(\sup _{t \geq M} \rho\left(f-h_{t}\right)\right)=\lim _{M \rightarrow \infty} \sup _{t \geq M} \rho\left(f-h_{t}\right)
$$

for any $f \in C$, we get

$$
\rho\left(\frac{f_{1}-f_{2}}{2}\right) \leq \tau\left(f_{1}\right)+\tau\left(f_{2}\right)=0,
$$

which implies $f_{1}=f_{2}$ as claimed.

Case 2: $\inf _{f \in C} \tau(f)>0$. Assume to the contrary that $f_{1} \neq f_{2}$. Set

$$
R=\inf _{f \in C} \tau(f) \quad \text { and } \quad \varepsilon=\frac{\rho\left(f_{1}-f_{2}\right)}{2 R} .
$$

Let $v \in(0, R)$. Then $\rho\left(f_{1}-f_{2}\right)=2 R \varepsilon \geq(R+v) \varepsilon$. Using the definition of $\tau$, we deduce that there exists $M_{v}>0$ such that

$$
\sup _{t \geq M_{\nu}} \rho\left(f_{1}-h_{t}\right) \leq \tau\left(f_{1}\right)+v=R+v \quad \text { and } \quad \sup _{t \geq M_{\nu}} \rho\left(f_{2}-h_{t}\right) \leq \tau\left(f_{2}\right)+v=R+v .
$$

Since $\rho$ is (UUC), there exists $\eta(R, \varepsilon)>0$ such that

$$
\delta(R+v, \varepsilon) \geq \eta(R, \varepsilon)
$$

for any $v \in(0, R)$. So for any $t \geq M_{v}$, we have

$$
\rho\left(\frac{f_{1}+f_{2}}{2}-h_{t}\right) \leq(R+v)(1-\delta(R+v, \varepsilon)) \leq(R+v)(1-\eta(R, \varepsilon)) .
$$

Hence

$$
\tau\left(\frac{f_{1}+f_{2}}{2}\right) \leq \sup _{t \geq M_{v}} \rho\left(\frac{f_{1}+f_{2}}{2}-h_{t}\right) \leq(R+v)(1-\eta(R, \varepsilon)) .
$$

Since $C$ is convex, we get

$$
R \leq \tau\left(\frac{f_{1}+f_{2}}{2}\right) \leq(R+\nu)(1-\eta(R, \varepsilon)) .
$$

If we let $v \rightarrow 0$, we will get

$$
R \leq R(1-\eta(R, \varepsilon))
$$

which is impossible, since $R>0$ and $\eta(R, \varepsilon)>0$. Therefore, we must have $f_{1}=f_{2}$.

Next, we prove (ii). Denote $R=\inf _{g \in C} \tau(g)$. For any $n \geq 1$, let us set

$$
K_{n}=\overline{\operatorname{conv}}_{\rho}\left\{h_{t} ; t \geq n\right\},
$$


where $\overline{\operatorname{conv}}_{\rho}(A)$ is the intersection of all $\rho$-closed convex subset of $C$, which contains $A \subset C$. Since $C$ is itself $\rho$-closed and convex, we get $K_{n} \subset C$ for any $n \geq 1$. Property $(R)$ will then imply $\bigcap K_{n} \neq \emptyset$. Let us fix then arbitrary $f \in \bigcap K_{n}, g \in C$ and $\varepsilon>0$. By definition of $\tau(g)$, there exists $M_{\varepsilon}>0$ such that $\sup _{t \geq M_{\varepsilon}} \rho\left(g-h_{t}\right) \leq \tau(g)+\varepsilon$. Let $n \geq M_{\varepsilon}$. Then for any $t \geq n$, we have $\rho\left(g-h_{t}\right) \leq \tau(g)+\varepsilon$, i.e., $h_{t} \in B_{\rho}(g, \tau(g)+\varepsilon)$. Since $B_{\rho}(g, \tau(g)+\varepsilon)$ is $\rho$-closed and convex, we get $K_{n} \subset B_{\rho}(g, \tau(g)+\varepsilon)$. Hence $f \in B_{\rho}(g, \tau(g)+\varepsilon)$, i.e.,

$$
\rho(g-f) \leq \tau(g)+\varepsilon
$$

Since $\varepsilon$ was taken arbitrarily greater than 0 , we get $\rho(g-f) \leq \tau(g)$ for any $g \in C$. Let $\left\{f_{n}\right\}$ be a minimizing sequence for $\tau$. If $R=0$, then, since $\left\{f_{n}\right\}$ is a minimizing sequence, we get $\lim _{n \rightarrow \infty} \tau\left(f_{n}\right)=R=0$. Using (3.1), we can see that $\rho\left(f_{n}-f\right) \leq \tau\left(f_{n}\right)$ for any $n \geq 1$. Hence $\left\{f_{n}\right\}$ is $\rho$-convergent to $f$. Since selection of $f$ was independent of $\left\{f_{n}\right\}$, it follows that any minimizing sequence is $\rho$-convergent to $f$ if $R=0$. We can assume, therefore, that $R>0$. For any $n \geq 1$, let us set

$$
d_{n}=\sup _{i, j \geq n} \rho\left(f_{i}-f_{j}\right) .
$$

We claim that $\left\{f_{n}\right\}$ is $\rho$-Cauchy. Assume to the contrary that this is not the case. Since the sequence $\left\{d_{n}\right\}$ is decreasing and $\left\{f_{n}\right\}$ is not $\rho$-Cauchy, we get $d:=\inf _{n \geq 1} d_{n}>0$. Set $\varepsilon=\frac{d}{4 R}>0$. Let us fix arbitrary $v \in(0, R)$. Since $\lim _{n \rightarrow \infty} \tau\left(f_{n}\right)=R$, there exists $n_{0} \geq 1$ such that for any $n \geq n_{0}$, we have

$$
\tau\left(f_{n}\right) \leq R+\frac{v}{2}
$$

Let $n \geq n_{0}$. By (3.2), there exists $i_{n}, j_{n} \geq 1$ such that

$$
\rho\left(f_{i_{n}}-f_{j_{n}}\right)>d_{n}-\frac{d}{2} \geq \frac{d}{2}=2 R \varepsilon>(R+v) \varepsilon .
$$

Using the definition of $\tau$ and (3.3), we deduce the existence of $M>0$ such that

$$
\sup _{t \geq M} \rho\left(f_{i_{n}}-h_{t}\right) \leq \tau\left(f_{i_{n}}\right)+\frac{v}{2} \leq R+v
$$

and

$$
\sup _{t \geq M} \rho\left(f_{j_{n}}-h_{t}\right) \leq \tau\left(f_{j_{n}}\right)+\frac{v}{2} \leq R+v .
$$

Hence

$$
\rho\left(\frac{f_{i_{n}}+f_{j_{n}}}{2}-h_{t}\right) \leq(R+v)(1-\delta(R+v, \varepsilon))
$$

for any $t \geq M$. Since $\rho$ is (UUC), there exists $\eta_{1}(R, \varepsilon)>0$ such that $\delta_{1}(R+v, \varepsilon) \geq \eta_{1}(R, \varepsilon)$. Hence

$$
\rho\left(\frac{f_{i_{n}}+f_{j_{n}}}{2}-h_{t}\right) \leq(R+v)(1-\eta(R, \varepsilon))
$$


for any $t \geq M$. Hence

$$
R \leq \tau\left(\frac{f_{i_{n}}+f_{j_{n}}}{2}\right) \leq \sup _{t \geq M} \rho\left(\frac{f_{j_{n}}+f_{j_{n}}}{2}-h_{t}\right) \leq(R+v)(1-\eta(R, \varepsilon))<R .
$$

Using the definition of $R$, we get

$$
R \leq(R+v)(1-\eta(R, \varepsilon))
$$

for any $v \in(0, R)$. If we let $v \rightarrow 0$, we get $R \leq R\left(1-\eta_{1}(R, \varepsilon)\right)$. This contradiction implies that $\left\{f_{n}\right\}$ is $\rho$-Cauchy. Since $L_{\rho}$ is $\rho$-complete, we deduce that $\left\{f_{n}\right\}$ is $\rho$-convergent as claimed.

In order to finish the proof of (ii), let us show that the $\rho$-limit of $\left\{f_{n}\right\}$ is independent of the minimizing sequence. Indeed, let $\left\{g_{n}\right\}$ be another minimizing sequence of $\tau$. The previous proof will show that $\left\{g_{n}\right\}$ is also $\rho$-convergent. In order to prove that the $\rho$-limits of $\left\{f_{n}\right\}$ and $\left\{g_{n}\right\}$ are equal, let us show that $\lim _{n \rightarrow \infty} \rho\left(f_{n}-g_{n}\right)=0$. Assume not, i.e., $\lim _{n \rightarrow \infty} \rho\left(f_{n}-g_{n}\right) \neq 0$. Without loss of generality, we may assume that there exists $d>0$ such that $\rho\left(f_{n}-g_{n}\right) \geq d$ for any $n \geq 1$. Set $\varepsilon=\frac{d}{2 R}>0$. Let $v \in(0, R)$. Since $\lim _{n \rightarrow \infty} \tau\left(f_{n}\right)=$ $\lim _{n \rightarrow \infty} \tau\left(g_{n}\right)=R$, there exists $n_{0} \geq 1$ such that for any $n \geq 1$, we have $\tau\left(f_{n}\right) \leq R+\frac{v}{2}$ and $\tau\left(g_{n}\right) \leq R+\frac{v}{2}$. Fix $n \geq n_{0}$. Then

$$
\rho\left(f_{n}-g_{n}\right) \geq d=2 R \varepsilon>(R+v) \varepsilon .
$$

Using the definition of $\tau$, we deduce the existence of $M>0$ such that

$$
\sup _{t \geq M} \rho\left(f_{n}-h_{t}\right) \leq \tau\left(f_{n}\right)+\frac{v}{2} \leq R+v
$$

and

$$
\sup _{t \geq M} \rho\left(g_{n}-h_{t}\right) \leq \tau\left(g_{n}\right)+\frac{v}{2} \leq R+v .
$$

Hence

$$
\rho\left(\frac{f_{n}+g_{n}}{2}-h_{t}\right) \leq(R+v)(1-\delta(R+v, \varepsilon))
$$

for any $t \geq M$. Since $\rho$ is (UUC), there exists $\eta(R, \varepsilon)>0$ such that $\delta(R+\nu, \varepsilon) \geq \eta(R, \varepsilon)$ for any $v>0$. Hence

$$
\rho\left(\frac{f_{n}+g_{n}}{2}-h_{t}\right) \leq(R+v)(1-\eta(R, \varepsilon))
$$

for any $t \geq M$. So

$$
\tau\left(\frac{f_{n}+g_{n}}{2}\right) \leq \sup _{t \geq M} \rho\left(\frac{f_{n}+g_{n}}{2}-h_{t}\right) \leq(R+v)(1-\eta(R, \varepsilon)) .
$$

Using the definition of $R$, we get

$$
R \leq(R+v)(1-\eta(R, \varepsilon))
$$


for any $v \in(0, R)$. If we let $v \rightarrow 0$, we get $R \leq R(1-\eta(R, \varepsilon))$. This contradiction implies $\lim _{n \rightarrow \infty} \rho\left(f_{n}-g_{n}\right)=0$. The Fatou property will finally imply that

$$
\rho(f-g) \leq \liminf _{n \rightarrow \infty} \rho\left(f_{n}-g_{n}\right)
$$

where $f$ is the $\rho$-limit of $\left\{f_{n}\right\}$ and $g$ is the $\rho$-limit of $\left\{g_{n}\right\}$. Hence $\rho(f-g)=0$, i.e., $f=g$.

Using Lemma 3.2, we are ready to prove our common fixed point result for asymptotic pointwise nonexpansive semigroups.

Theorem 3.2 Assume $\rho \in \Re$ is $(U U C)$. Let $C$ be a $\rho$-closed, $\rho$-bounded convex nonempty subset. Let $\mathcal{F}=\left\{T_{t}: t \geq 0\right\}$ be an asymptotic pointwise nonexpansive semigroup on $C$. Then $\mathcal{F}$ has a common fixed point, and the set $F(\mathcal{F})$ of common fixed points is $\rho$-closed and convex.

Proof Let us fix $f \in C$ and define the function

$$
\tau(g)=\inf _{M>0}\left(\sup _{t \geq M} \rho\left(T_{t}(f)-g\right)\right) .
$$

Since $C$ is $\rho$-bounded, we have $\tau(g) \leq \operatorname{diam}_{\rho}(C)<+\infty$ for any $g \in C$. Hence $\tau_{0}=\inf \{\tau(g)$ : $g \in C$ \} exists and is finite. For any $n \geq 1$, there exists $g_{n} \in C$, such that

$$
\tau_{0} \leq \tau\left(g_{n}\right)<\tau_{0}+\frac{1}{n}
$$

Therefore, $\lim _{n \rightarrow \infty} \tau\left(z_{n}\right)=\tau_{0}$, i.e., $\left\{g_{n}\right\}$ is a minimizing sequence for $\tau$. By Lemma 3.2, there exists $g \in C$ such that $\left\{g_{n}\right\} \rho$-converges to $g$. Let us now prove that $g \in F(\mathcal{F})$. Note that

$$
\rho\left(T_{s+t}(f)-T_{s}(h)\right) \leq \alpha_{s}(h) \rho\left(T_{t}(f)-h\right)
$$

for $s, t>0$ and $h \in C$. Using the definition of $\tau$, we get

$$
\tau\left(T_{s}(h)\right) \leq \sup _{t+s \geq M} \rho\left(T_{s+t}(f)-T_{s}(h)\right) \leq \alpha_{s}(h) \sup _{t \geq M-s} \rho\left(T_{t}(f)-h\right)
$$

for any $M>s$, which implies

$$
\tau\left(T_{s}(h)\right) \leq \alpha_{s}(h) \tau(h)
$$

Since $\lim _{s \rightarrow \infty} \alpha_{s}\left(g_{1}\right)=1$, there exists $s_{1}>0$ such that for any $s \geq s_{1}$, we have $\alpha_{s}\left(g_{1}\right)<1+1$. Repeating this argument, one will find $s_{2}>s_{1}+1$ such that for any $s \geq s_{2}$, we have $\alpha_{s}\left(g_{2}\right)<$ $1+\frac{1}{2}$. By induction, we will construct a sequence $\left\{s_{n}\right\}$ of positive numbers such that $s_{n+1}<$ $s_{n}+\frac{1}{n}$ and for any $s \geq s_{n}$, we have $\alpha_{s}\left(g_{n}\right)<1+\frac{1}{n}$. Let us fix $t \geq 0$. Then inequality (3.4) will imply

$$
\tau\left(T_{s_{n}+t}\left(g_{n}\right)\right) \leq \alpha_{s_{n}+t}\left(g_{n}\right) \tau\left(g_{n}\right) \leq\left(1+\frac{1}{n}\right) \tau\left(g_{n}\right)
$$


for any $n \geq 1$. In particular, we get that $\left\{T_{s_{n}+t}\left(g_{n}\right)\right\}$ is a minimizing sequence of $\tau$. Therefore, Lemma 3.2 implies that $\left\{T_{s_{n}+t}\left(g_{n}\right)\right\} \rho$-converges to $g$ for any $t \geq 0$. In particular, we have $\left\{T_{s_{n}}\left(g_{n}\right)\right\} \rho$-converges to $g$. Since

$$
\rho\left(T_{s_{n}+t}\left(g_{n}\right)-T_{t}(g)\right) \leq \alpha_{t}(g) \rho\left(T_{s_{n}}\left(g_{n}\right)-g\right),
$$

we get $\left\{T_{s_{n}+t}\left(g_{n}\right)\right\} \rho$-converges to $T_{t}(g)$. Finally, using

$$
\rho\left(\frac{T_{t}(g)-g}{2}\right) \leq \rho\left(T_{t}(g)-T_{s_{n}+t}\left(g_{n}\right)\right)+\rho\left(T_{s_{n}+t}\left(g_{n}\right)-g\right)
$$

we get $T_{t}(g)=g$. Since $t$ was arbitrarily positive, we get $g \in F(\mathcal{F})$, i.e., $F(\mathcal{F})$ is not empty. Next, let us prove that $F(\mathcal{F})$ is $\rho$-closed. Let $\left\{f_{n}\right\}$ in $F(\mathcal{F}) \rho$-convergent to $f$. Since

$$
\rho\left(T_{s}\left(f_{n}\right)-T_{s}(f)\right) \leq \alpha_{s}(f) \rho\left(f_{n}-f\right)
$$

for any $n \geq 1$ and $s>0$, we get $\left\{T_{s}\left(f_{n}\right)\right\} \rho$-converges to $T_{s}(f)$. Since $f_{n} \in F(\mathcal{F})$, we get $\left\{T_{s}\left(f_{n}\right)\right\}=\left\{f_{n}\right\}$. In other words, $\left\{f_{n}\right\} \rho$-converges to $T_{s}(f)$ and $f$. The uniqueness of the $\rho$-limit implies then that $T_{s}(f)=f$ for any $s \geq 0$, i.e., $f \in F(\mathcal{F})$. Therefore, $F(\mathcal{F})$ is $\rho$-closed. Let us finish the proof of Theorem 3.2 by showing that $F(\mathcal{F})$ is convex. It is sufficient to show that

$$
h=\frac{f+g}{2} \in F(\mathcal{F})
$$

for any $f, g \in F(\mathcal{F})$. Without loss of generality, we will assume that $f \neq g$. Let $s>0$. We have

$$
\rho\left(f-T_{s}(h)\right)=\rho\left(T_{s}(f)-T_{s}(h)\right) \leq \alpha_{s}(f) \rho(f-h)
$$

and

$$
\rho\left(g-T_{s}(h)\right)=\rho\left(T_{s}(g)-T_{s}(h)\right) \leq \alpha_{s}(g) \rho(g-h) .
$$

Since $\rho(f-h)=\rho(g-h)=\rho\left(\frac{f-g}{2}\right)$ and

$$
\rho\left(\frac{f-g}{2}\right) \leq \frac{1}{2} \rho\left(f-T_{s}(h)\right)+\frac{1}{2} \rho\left(g-T_{s}(h)\right)
$$

we conclude that

$$
\lim _{s \rightarrow \infty} \rho\left(f-T_{s}(h)\right)=\lim _{s \rightarrow \infty} \rho\left(g-T_{s}(h)\right)=\rho\left(\frac{f-g}{2}\right) .
$$

Similarly, we have

$$
\rho\left(f-\frac{h+T_{s}(h)}{2}\right) \leq \frac{1}{2} \rho(f-h)+\frac{1}{2} \rho\left(f-T_{s}(h)\right)
$$

and

$$
\rho\left(g-\frac{h+T_{s}(h)}{2}\right) \leq \frac{1}{2} \rho(g-h)+\frac{1}{2} \rho\left(g-T_{s}(h)\right) .
$$


Since

$$
\rho\left(\frac{f-g}{2}\right) \leq \frac{1}{2} \rho\left(f-\frac{h+T_{s}(h)}{2}\right)+\frac{1}{2} \rho\left(g-\frac{h+T_{s}(h)}{2}\right),
$$

we conclude that

$$
\lim _{s \rightarrow \infty} \rho\left(f-\frac{h+T_{s}(h)}{2}\right)=\lim _{s \rightarrow \infty} \rho\left(g-\frac{h+T_{s}(h)}{2}\right)=\rho\left(\frac{f-g}{2}\right) .
$$

Therefore, we have

$$
\lim _{s \rightarrow \infty} \rho\left(f-T_{s}(h)\right)=\lim _{s \rightarrow \infty} \rho\left(f-\frac{h+T_{s}(h)}{2}\right)=\rho(f-h) .
$$

Lemma 3.1, applied to $A_{t}=f-T_{s}(h)$ and $B_{t}=T_{s}(h)-g$, implies that $\rho\left(A_{t}-B_{t}\right) \rightarrow 0$. Hence

$$
\lim _{s \rightarrow \infty} \rho\left(h-T_{s}(h)\right)=\lim _{s \rightarrow \infty} \rho\left(\frac{A_{t}-B_{t}}{2}\right) \leq \lim _{s \rightarrow \infty} \rho\left(A_{t}-B_{t}\right)=0 .
$$

Clearly, we will get $\lim _{s \rightarrow \infty} \rho\left(h-T_{s+t}(h)\right)=0$, for any $t \geq 0$. Since

$$
\rho\left(T_{t}(h)-T_{s+t}(h)\right) \leq \alpha_{t}(h) \rho\left(h-T_{s}(h)\right),
$$

we get $\lim _{s \rightarrow \infty} \rho\left(T_{t}(h)-T_{s+t}(h)\right)=0$. Finally, using the inequality

$$
\rho\left(\frac{h-T_{t}(h)}{2}\right) \leq \frac{1}{2} \rho\left(h-T_{s+t}(h)\right)+\frac{1}{2} \rho\left(T_{t}(h)-T_{s+t}(h)\right),
$$

by letting $s \rightarrow \infty$, we get $T_{t}(h)=h$ for any $t \geq 0$, i.e., $h \in F(\mathcal{F})$.

\section{Competing interests}

The authors declare that they have no competing interests.

\section{Authors' contributions}

All authors participated in the design of this work and performed equally. All authors read and approved the final manuscript.

\section{Author details}

${ }^{1}$ Department of Mathematics, King Abdulaziz University, P.O. Box 53909, Jeddah, 21593, Saudi Arabia. ${ }^{2}$ Department of Mathematical Sciences, University of Texas at El Paso, El Paso, Texas, USA. ${ }^{3}$ Department of Mathematics and Statistics, King Fahd University of Petroleum \& Minerals, Dhahran, 31261, Saudi Arabia. ${ }^{4}$ School of Mathematics and Statistics, University of New South Wales, Sydney, NSW 2052, Australia.

\section{Acknowledgements}

This work was funded by the Deanship of Scientific Research (DSR), King Abdulaziz University, Jeddah, under Grant No. (247-006-D1433). The authors, therefore, acknowledge with thanks technical and financial support of DSR.

\section{Received: 28 February 2013 Accepted: 24 July 2013 Published: 12 August 2013}

\section{References}

1. Kozlowski, WM: Modular Function Spaces. Series of Monographs and Textbooks in Pure and Applied Mathematics, vol. 122. Dekker, New York (1988)

2. Khamsi, MA, Kozlowski, WM, Reich, S: Fixed point theory in modular function spaces. Nonlinear Anal. 14, 935-953 (1990)

3. Khamsi, MA, Kozlowski, WM, Shutao, C: Some geometrical properties and fixed point theorems in Orlicz spaces. J. Math. Anal. Appl. 155(2), 393-412 (1991) 
4. Khamsi, MA: Fixed point theory in modular function spaces. In: Proceedings of the Workshop on Recent Advances on Metric Fixed Point Theory, Sevilla, September 1995, pp. 31-35 (1996). MR1440218 (97m:46044)

5. Kozlowski, WM: Advancements in fixed point theory in modular function. Arab. J. Math. (2012). doi:10.1007/s40065-012-0051-0

6. Khamsi, MA, Kozlowski, WM: On asymptotic pointwise contractions in modular function spaces. Nonlinear Anal. 73, 2957-2967 (2010)

7. Khamsi, MA, Kozlowski, WM: On asymptotic pointwise nonexpansive mappings in modular function spaces. J. Math. Anal. Appl. 380(2), 697-708 (2011)

8. Belluce, LP, Kirk, WA: Fixed-point theorems for families of contraction mappings. Pac. J. Math. 18, $213-217$ (1966)

9. Belluce, LP, Kirk, WA: Nonexpansive mappings and fixed-points in Banach spaces. III. J. Math. 11, 474-479 (1967)

10. Browder, FE: Nonexpansive nonlinear operators in a Banach space. Proc. Natl. Acad. Sci. USA 54, 1041-1044 (1965)

11. Bruck, RE: A common fixed point theorem for a commuting family of nonexpansive mappings. Pac. J. Math. 53, 59-71 (1974)

12. DeMarr, RE: Common fixed-points for commuting contraction mappings. Pac. J. Math. 13, 1139-1141 (1963)

13. Lim, TC: A fixed point theorem for families of nonexpansive mappings. Pac. J. Math. 53, 487-493 (1974)

14. Tan, K-K, Xu, H-K: An ergodic theorem for nonlinear semigroups of Lipschitzian mappings in Banach spaces. Nonlinear Anal. 19(9), 805-813 (1992)

15. Kirk, WA, Xu, HK: Asymptotic pointwise contractions. Nonlinear Anal. 69, 4706-4712 (2008)

16. Hussain, N, Khamsi, MA: On asymptotic pointwise contractions in metric spaces. Nonlinear Anal. 71(10), 4423-4429 (2009)

17. Kozlowski, WM: Fixed point iteration processes for asymptotic pointwise nonexpansive mappings in Banach spaces. J. Math. Anal. Appl. 377(1), 43-52 (2011)

18. Kozlowski, WM: Pointwise Lipschitzian mappings in uniformly convex and uniformly smooth Banach spaces. Nonlinear Anal. (2013). doi:10.1016/j.na.2013.02.003

19. Kozlowski, WM: Common fixed points for semigroups of pointwise Lipschitzian mappings in Banach spaces. Bull. Aust. Math. Soc. 84, 353-361 (2011)

20. Kozlowski, WM, Sims, B: On the convergence of iteration processes for semigroups of nonlinear mappings in Banach spaces (to appear)

21. Kozlowski, WM: On the construction of common fixed points for semigroups of nonlinear mappings in uniformly convex and uniformly smooth Banach spaces. Comment. Math. 52(2), 113-136 (2012)

22. Bin Dehaish, BA, Kozlowski, WM: Fixed point iterations processes for asymptotic pointwise nonexpansive mappings in modular function spaces. Fixed Point Theory Appl. 2012, 118 (2012)

23. Kozlowski, WM: On the existence of common fixed points for semigroups of nonlinear mappings in modula function spaces. Comment. Math. 51(1), 81-98 (2011)

24. Khamsi, MA: Nonlinear semigroups in modular function spaces. Math. Jpn. 37(2), 1-9 (1992)

25. Kozlowski, WM: Notes on modular function spaces I. Comment. Math. 28, 91-104 (1988)

26. Kozlowski, WM: Notes on modular function spaces II. Comment. Math. 28, 105-120 (1988)

doi:10.1186/1687-1812-2013-214

Cite this article as: Bin Dehaish et al.: Common fixed points for pointwise Lipschitzian semigroups in modular function spaces. Fixed Point Theory and Applications 2013 2013:214.

\section{Submit your manuscript to a SpringerOpen ${ }^{\ominus}$ journal and benefit from:}

- Convenient online submission

Rigorous peer review

- Immediate publication on acceptance

- Open access: articles freely available online

- High visibility within the field

- Retaining the copyright to your article 